\title{
Entrenamiento combinado de fuerza y ejercicios de saltos, efectos sobre el rendimiento en el salto vertical en un grupo de alto nivel de jugadores de voleibol durante una temporada completa de competición

\author{
Combined strength and jump exercises training, effects on the vertical jump performance in a group of \\ senior elite male volleyball players during a complete competition season \\ *Fundación CajaSol-JUVASA Voleibol Esquimo SVM, **Universidad Pablo de Olavide
} \\ Carlos García Asencio*, Miguel Sánchez Moreno*, Juan José González Badillo**
}

\begin{abstract}
Resumen. Se ha sugerido que para mejorar el rendimiento en el salto vertical (SV), los jugadores de voleibol deben realizar entrenamiento con cargas específico para voleibol. Este estudio examina los efectos del entrenamiento combinado de fuerza y ejercicios de saltos sobre el rendimiento en el SV, en un grupo de alto nivel de jugadores de voleibol durante una temporada completa de la competición. Doce jugadores profesionales de voleibol participaron en este estudio. El rendimiento se midió mediante la altura (cm) del salto sin carga (CMJ) y con carga (CMJ ${ }_{\text {loaded }}$ ), y por la velocidad de desplazamiento en fase concéntrica de la sentadilla completa (FS) (m/s). El entrenamiento tuvo lugar 2 días/semana, 16 semanas (2 ciclos / 8 semanas, 1 semana de descanso). Los ejercicios de resistencia fueron: FS, 3-4 series / 4-6 repeticiones, 50\% al 65\% de 1RM; cargada de fuerza, 3 series / 4-6 repeticiones, con máxima carga que permite ejecución técnica correcta; saltos con carga, 3-4 series / 4-5 repeticiones, 50\%-80\% carga con la que alcanzaron una altura de unos 20 cm.; salto sin carga, 3-4 series / 5 repeticiones. Hubo un aumento significativo en CMJ y CMJloaded (7.12\% y 9.78\%; $\mathrm{p}<.01$; respectivamente). No se observaron cambios significativos en FS (1.66\%; $>>.05)$. Nuestros resultados sugieren que el uso de cargas moderadas podría producir resultados similares en el rendimiento del SV al uso de cargas elevadas, ya que en nuestro estudio no superamos una carga equivalente al $65 \%$ de 1RM en el FS y los ejercicios de salto se realizaron con cargas ligeras. Estos resultados indican que la especificidad del entrenamiento, expresada por la proximidad de las velocidades de ejecución de los ejercicios de entrenamiento a la velocidad de ejecución del SV, es determinante para el rendimiento.
\end{abstract}

Palabras clave. Voleibol, entrenamiento combinado, salto vertical, entrenamiento específico con cargas.

Abstrac. It has been suggested that to improve vertical jump performance, volleyball players must complete specific volleyball resistance training. This study examines the effects of combined strength and jump exercises training on the vertical jump performance, in a group of senior elite male volleyball players during a full competition season. Twelve professional male volleyball players took part in this study. Players competed in the first national division of the Spanish National League during the 2014-2015 season. The performance was estimated by unloaded (CMJ), loaded countermovement jumps (CMJ ) height $(\mathrm{cm})$ and by velocity of displacement in the concentric phase of full squat $(\mathrm{FS})\left(\mathrm{m} \bullet \mathrm{s}^{-1}\right)$. Training took place 2 days/week, 16 weeks (2 cycles/8 weeks, 1 week recovery). The resistance exercises were: FS, 3-4 series/4-6 repetitions, load from 50\%-65\% 1RM; hang power clean, 3 series/4-6 repetitions with the maximum load that allows a correct technical execution; loaded jump, 3-4 series/4-5 repetitions, load from 50\%-80\% according to the weight with which the athletes reached a height of about $20 \mathrm{~cm}$.; unloaded jump, 3-4 series/5 repetitions. There was a significant increase in CMJ and CMJloaded (7.12\% and 9.78\%; $\mathrm{p}<.01$; respectively). No significant changes were observed in FS (1.66\%; $>>.05)$. Our results suggest that the use of moderate loads could produce similar results on vertical jump performance to the use of high loads, since in our study a load equivalent to $65 \% 1 \mathrm{RM}$ in the FS was not exceeded and jumping exercises were performed with light loads. These results indicate that the specificity of training, which in this case is expressed by the proximity of the velocities of execution of the training exercises to the velocity of execution of the vertical jump, is determinant for the performance.

Keywords. Volleyball, combined training, vertical jump, specific resistance training.

\section{Introducción}

El voleibol es uno de los deportes más populares del mundo, y se caracteriza por la exigencia de realizar movimientos comúnmente conocidos como explosivos, tales como saltos, golpeos y desplazamientos cortos. Además de unas adecuadas habilidades técnicas y tácticas, unas características morfológicas apropiadas (Lidor \& Ziv, 2010) y una fuerza muscular bien desarrollada (Häkkinen, 1993; Marques, Van Den Tillaar, Vescovi, \& González-Badillo, 2008; Sheppard, Chapman, Gough, McGuigan, \& Newton, 2009), se ha sugerido que la capacidad de salto vertical es uno de los elementos claves para alcanzar el éxito en la práctica del voleibol (Forthomme, Croisier, Ciccarone, Crielaard, \& Cloes, 2005; Stanganelli, Dourado, Oncken, Mançan, \& da Costa, 2008). Las diferencias observadas en el rendimiento en el salto vertical (SV) entre jugadores de voleibol de distinto nivel competitivo (Forthomme et al., 2005; Fry et al., 1991) ilustran la importancia de dicha habilidad dentro de esta disciplina deportiva.

Algunos autores sugieren que un programa de entrenamiento basado exclusivamente en el uso de habilidades específicas de voleibol puede no ser suficiente para la mejora del salto vertical en este tipo de deportistas, y que la combinación de este con un programa de entrenamiento de fuerza podría ser la forma más adecuada para el desarrollo de este tipo de deportistas (Trajkovic, Milanovic, Sporis, Milic, \& Stankovic,

Fecha recepción: 07-07-15- Fecha envío revisores: 07-07-15- Fecha de aceptación: 23-11-15 Carlos Garća Asencio asencio9@gmail.com
2012). Sin embargo, la información disponible en la literatura acerca de los efectos de un entrenamiento de fuerza sobre el rendimiento en SV en jugadores de voleibol durante un periodo competitivo es escasa (González-Ravé, Arija, \& Clemente-Suarez, 2011; Häkkinen, 1993; Marques et al., 2008; Sánchez-Moreno, García-Asencio, \& GonzálezBadillo, 2014; Stanganelli et al., 2008). Estudios previos realizados con jugadoras de elite de voleibol han utilizado entrenamientos de fuerza empleando cargas altas o pesadas (>70\% de una repetición máxima [1RM]) (Häkkinen, 1993), o una combinación de cargas altas y ejercicios pliométricos (González-Ravé et al., 2011; Marques et al., 2008) con el objetivo de mejorar las características de fuerza y potencia en este tipo de deportistas. En estas investigaciones se observaron efectos positivos tras la aplicación de estos tipos de entrenamiento, dando lugar a incrementos significativos en el SV, sin embargo, otros autores (Voelzke, Stutzig, Thorhauer, \& Granacher, 2012) observaron una falta de mejora en el SV tras el uso de entrenamiento de fuerza con cargas pesadas (85\%1RM).

Entrenamientos de resistencias que emplean cargas pesadas y repeticiones al fallo o, cerca de este, están asociados con grados de fatiga altos (Luis Sanchez-Medina \& González-Badillo, 2011). Algunos autores sugieren que no es necesario causar una fatiga excesiva para la mejora de la fuerza (González-Badillo et al., 2015; Izquierdo et al., 2006) y que la velocidad de levantamientos es más o, al menos, igual de importante como la magnitud de la carga en sí mismo (Pareja-Blanco, Rodríguez-Rosell, Sánchez-Medina, Gorostiaga, \& González-Badillo, 2014).

El uso de la velocidad de movimiento como referencia para programar el entrenamiento de fuerza es un método novedoso para medir la 
fuerza de los atletas sin necesidad de realizar un test de 1RM o un test de repeticiones máximas (XRM). Esto es posible debido a la estrecha relación que existe $\left(\mathrm{R}^{2}=.98\right)$ entre la velocidad de movimiento y la carga relativa (\%1RM) (González-Badillo \& Sánchez-Medina, 2010). Según nuestro conocimiento, pocos estudios previos (Franco-Márquez et al., 2015; González-Badillo et al., 2015; López-Segovia, Andrés, \& González-Badillo, 2010; Pareja-Blanco etal., 2014; Sáez Sáez de Villarreal, Izquierdo, \& Gonzalez Badillo, 2011; Sánchez-Moreno et al., 2014) han usado la velocidad de movimiento para prescribir el entrenamiento de fuerza, de los cuales, solo uno de ellos fue realizado con jugadores de elite de voleibol (Sánchez-Moreno et al., 2014).

Por tanto, el objetivo de este estudio fue examinar los efectos de un entrenamiento de fuerza realizado durante la temporada de competición caracterizado por el uso de cargas moderadas y un numero de repeticiones bajo por serie combiando con ejercicios pliométricos sobre la capacidad de SV, saltos con cargas, y la fuerza del miembro inferior en jugadores de elite de voleibol.

\section{Material y método}

\section{Muestra}

12 jugadores varones ( $\mathrm{n}=12$; edad [años]: $27.7 \pm 4.5$; altura: $1.86 \pm$ .06 m.; peso corporal: $78.8 \pm 7.04 \mathrm{~kg}$.) pertenecientes a un equipo que militaba en la primera liga nacional española, los cuales no mostraron ningún tipo de lesión o limitación musculo esquelética que pudiera afectar a los test, participaron en este estudio. Todos los jugadores fueron informados sobre los objetivos y factores de riesgo de este estudio, y estuvieron de acuerdo a participar en él después de explicar el proceso experimental.

\section{Protocolo de entrenamiento}

El entrenamiento de fuerza consistió en 2 sesiones de entrenamiento a la semana (martes y jueves) con una duración de aproximadamente 45 minutos. Todos los entrenamientos fueron realizados previos al entrenamiento técnico-táctico y fueron supervisados por un miembro del grupo de investigación.

Los ejercicios de entrenamientos fueron: sentadilla completa (FS), 3-4 series / 4-6 repeticiones, con una carga del 50\% al 65\% de 1RM; cargada de fuerza, 3 series / 4-6 repeticiones, con la máxima carga que permitía una ejecución técnica correcta; saltos con carga, 3-4 series / 45 repeticiones, con $50 \%-80 \%$ de la carga del con la que alcanzaron una altura de unos 20 cm.; salto sin carga, 3-4 series / 5 repeticiones.

$$
\text { Tabla } 1 .
$$

\begin{tabular}{|c|c|c|c|c|c|}
\hline \multirow{2}{*}{$\begin{array}{c}\text { Ciclo } 1 \\
\text { Sem. }\end{array}$} & \multicolumn{5}{|c|}{ Ejercicios de entrenamiento } \\
\hline & $\mathrm{s} / \mathrm{s}$ & Sentadilla (FS) & Saltos con carga & Power clean & Saltos s.c. \\
\hline $0-1$ & 2 & $4 \times 6(1 \mathrm{~m} / \mathrm{s}-15 \%) / 3 \times 6(1 \mathrm{~m} / \mathrm{s}-15 \%)$ & $4 \times 5(50 \%) / 3 \times 5(50 \%)$ & $3 \times 6$ & $4 \times 5 / 3 \times 5$ \\
\hline 2 & 2 & $4 \times 5(1 \mathrm{~m} / \mathrm{s}-7 \%) / 3 \times 5(1 \mathrm{~m} / \mathrm{s}-7 \%)$ & $4 \times 5(60 \%) / 3 \times 5(60 \%)$ & $3 \times 6$ & $4 \times 5 / 3 \times 5$ \\
\hline 3 & 2 & $4 \times 6(1 \mathrm{~m} / \mathrm{s}-7 \%)^{* *}$ & $4 \times 5(60 \%)^{* * *}$ & $3 \times 6$ & $4 \times 5 * *$ \\
\hline 4 & 2 & $4 \times 4(1 \mathrm{~m} / \mathrm{s}) / 4 \times 5(1 \mathrm{~m} / \mathrm{s})$ & $4 \times 4(70 \%) / 4 \times 5(70 \%)$ & $3 \times 6$ & $4 \times 4 / 4 \times 5$ \\
\hline 5 & 2 & $4 \times 6(1 \mathrm{~m} / \mathrm{s})^{* *}$ & $4 \times 5(70 \%)^{* *}$ & $3 \times 5$ & $4 \times 5 * *$ \\
\hline 6 & 2 & $4 \times 4(1 \mathrm{~m} / \mathrm{s}+7 \%) / 3 \times 4(1 \mathrm{~m} / \mathrm{s}+7 \%)$ & $4 \times 4(80 \%) / 3 \times 4(80 \%)$ & $3 \times 5$ & $4 \times 5 / 3 \times 5$ \\
\hline $7-8$ & 2 & $4 \times 4(1 \mathrm{~m} / \mathrm{s}+7 \%)^{* * *}$ & $4 \times 4(80 \%)^{* * *}$ & $3 \times 5$ & $4 \times 5 * *$ \\
\hline Ciclo 2 & \multicolumn{5}{|c|}{ Ejercicios de entrenamiento } \\
\hline Sem. & $\mathrm{s} / \mathrm{s}$ & Sentadilla (FS) & Saltos con carga & Power clean & Saltos s.c. \\
\hline 0 & 2 & $4 \times 6(1 \mathrm{~m} / \mathrm{s}-15 \%) / 3 \times 6(1 \mathrm{~m} / \mathrm{s}-15 \%)$ & $4 \times 5(50 \%) / 3 \times 5(50 \%)$ & $3 \times 5$ & $3 \times 5 * *$ \\
\hline $1-2$ & 2 & $4 \times 6(1 \mathrm{~m} / \mathrm{s}-15 \%) / 3 \times 6$ (1m/s-15\%) & $4 \times 4(50 \%) / 3 \times 4(50 \%)$ & $3 \times 5$ & $3 \times 5^{* *}$ \\
\hline 3 & 2 & $4 \times 6(1 \mathrm{~m} / \mathrm{s}-7 \%) / 4 \times 6(1 \mathrm{~m} / \mathrm{s}-7 \%)$ & $4 \times 4(60 \%) * *$ & $3 \times 5$ & $3 \times 5^{* *}$ \\
\hline 4 & 2 & $4 \times 5(1 \mathrm{~m} / \mathrm{s}-7 \%) / 3 \times 5(1 \mathrm{~m} / \mathrm{s}-7 \%)$ & $4 \times 4(60 \%) / 3 \times 4(60 \%)$ & $3 \times 4$ & $3 \times 5 * *$ \\
\hline $5-6$ & 2 & $4 \times 5(1 \mathrm{~m} / \mathrm{s}) / 3 \times 5(1 \mathrm{~m} / \mathrm{s})$ & $4 \times 4(70 \%) / 3 \times 4(70 \%)$ & $3 \times 4$ & $3 \times 5^{* *}$ \\
\hline $7-8$ & 2 & $4 \times 4(1 \mathrm{~m} / \mathrm{s}+7 \%) / 3 \times 4(1 \mathrm{~m} / \mathrm{s}+7 \%)$ & $4 \times 4(80 \%) / 3 \times 4(80 \%)$ & $3 \times 3$ & $3 \times(2 \times 5) / 3 \times(2 \times 5)$ \\
\hline
\end{tabular}
a velocidad progresiva. s).

2, Diciembre (tras 8 semanas); y test 3, Marzo (tras 8 semanas), con una semana de descanso después de cada evaluación. Todos los test se realizaron tras un periodo de 48 horas de descanso completo después de la última competición, a la misma hora del día, y por los mismos investigadores. Todos los participantes fueron familiarizados con el instrumental utilizado y los protocolos del test mediante 2-3 intentos submáximos. Se realizó un calentamiento general que consistía en 5 minutos de carrera continua seguido de 5 series de 30 metros corriendo

El rendimiento se midió mediante la altura (cm) del salto sin carga (CMJ) y salto con carga $\left(\mathrm{CMJ}_{\text {loaded }}\right)$, y por la velocidad media propulsiva (VMP) (L Sanchez-Medina, Perez, \& Gonzalez-Badillo, 2010) del desplazamiento en la fase concéntrica de la sentadilla completa (FS) (m/

Test de Counter movement jump (CMJ). El CMJ se realizo utilizando dos zócalos de infrarrojos Optojump (Optojump, Microgate, Bolzano, Italia) para cronometrar el tiempo de vuelo. La altura del salto (h) se obtuvo a través de la fórmula: $\mathrm{h}=\mathrm{t}^{2} \bullet \mathrm{g} / \mathrm{g}$, donde $\mathrm{t}$ es el tiempo $\mathrm{y}$ g es la aceleración de la gravedad. Los deportistas realizaron 3 saltos con un descanso de 2 minutos entre cada salto. El valor medio de los tres saltos fue utilizado en el análisis.

Test de saltos con cargas ( $\left.C M J_{\text {loaded }}\right)$. El test consiste en realizar un salto vertical colocando la barra de pesas por detrás de la cabeza apoyada sobre la parte superior de la espalda. Para ello se utilizó una máquinapórtico tipo Smith machine (20kg), la cual se cargada progresivamente con aumentos de 5, 10 y $20 \mathrm{~kg}$ en cada repetición, hasta que el sujeto alcanzaba una altura en el salto aproximadamente $20 \mathrm{~cm}$, realizándose un descanso de 3 minutos entre cada repetición. Se controló estrictamente que no se despegase la barra del cuello al final del recorrido concéntrico, ya que esto podría proporcionar errores de medición. La altura del salto fue determinada del mismo modo que en el test CMJ.

Test de sentadillas. Dicho test se realizó en la misma máquina que el $\mathrm{CMJ}_{\text {loaded }}$, colocando la barra de pesas por detrás de la cabeza apoyada sobre la parte superior de la espalda. A partir de esta posición, se realizó una flexión profunda de las piernas, hasta sobrepasar la horizontal del muslo con respecto al suelo, pasando a continuación a la extensión inmediata de las piernas hasta la completa extensión. Los sujetos fueros instruidos para realizar una fase descendente controlada y una fase ascendente a la mayor velocidad posible. Después del calentamiento, la barra se fue cargando progresivamente, desde una velocidad media en la fase propulsiva (20) del movimiento aproximada de $1.4 \mathrm{~m} / \mathrm{s}$, con aumentos de 10 o $20 \mathrm{~kg}$ en cada serie, hasta que la velocidad media en la fase propulsiva del movimiento fue aproximadamente de $.95 \mathrm{~m} / \mathrm{s}$. Los sujetos realizaron entre 1 y 4 repeticiones con cada carga siendo registrada aquella que tuviese el mejor resultado acompañado de una ejecución correcta. Se realizó un descanso de 3 minutos entre cada carga. Utilizamos un dinamómetro isoinercial T-Force (T-Force System, Ergotech, Muria, España) para la medición de la velocidad del desplazamiento $(1.000 \mathrm{~Hz})$.

Análisis estadístico

Un análisis de la varianza (ANOVA para medidas repetidas, $\mathrm{p}<.05)$ se utilizó para medir los cambios en el CMJ, $\mathrm{CMJ}_{\text {loaded }} \mathrm{y}$ sentadillas entre los distintos test. El cálculo del tamaño del efecto (ES) fue realizado según Hedges y Olkin's (1985): $\mathrm{g}=\left(\mathrm{M}_{\text {post }}-\mathrm{M}_{\text {pre }}\right) / \mathrm{SD}_{\text {pooled' }}$, donde $\mathrm{M}_{\text {post }}$ es la media del post test, $\mathrm{M}_{\text {pre }}$ es la media del pretest y $\mathrm{SD}_{\text {pooled }}$ es la desviación típica agrupada de las medidas. La escala usada para la interpretación del ES fue la propuesta Rhea

\section{Test realizados e instrumental}

Todos los deportistas fueron evaluados en tres ocasiones: test 1 , Octubre (semana previa a comenzar los entrenamientos de fuerza); test
(2004) para deportistas altamente entrenados: ES trivial (<.25), pequeño (.25-.5), moderado $(.5-1,0)$ y grande $(>1.0)$. 


\section{Resultados}

Tuvo lugar un incremento significativo en el CMJ al comparar los resultados del test 1 con el test $2(5.3 \%, \mathrm{p}<.01)$ y del test 1 con el test $3(7.1 \%, \mathrm{p}<.01)$; dichos cambios fueron acompañados por valores del tamaño del efecto (ES) pequeño (.44) al comparar el test 1 y el test 2, y moderado (.58) al comparar el test 1 y el test 3 . En el caso del CMJ tuvo lugar un incremento significativo al comparar los resultados del test 2 con el test $3(5.7 \%, \mathrm{p}<.05)$ y del test 1 con el test $3(9.8 \%, \mathrm{p}<.01)$; dichos cambios fueron acompañados por valores del tamaño del efecto (ES) moderado (.82) al comparar el test 2 y el test 3, y grande (1.64) al comparar el test $1 \mathrm{y}$ el test 3 . No se observaron cambios significativos en el test de sentadilla completa (FS).

Tabla 2.

Resultados

\begin{tabular}{cccccccccc}
\hline & \multicolumn{3}{c}{ T1-T2 } & \multicolumn{3}{c}{ T2-T3 } & \multicolumn{3}{c}{ T1-T3 } \\
\hline Ejercicios & $\%$ & $\mathrm{p}$ & $\mathrm{Es}$ & $\%$ & $\mathrm{p}$ & $\mathrm{Es}$ & $\%$ & $\mathrm{p}$ & $\mathrm{Es}$ \\
\hline $\mathrm{CMJ}$ & 5.3 & .007 & .44 & 1.9 & .396 & .15 & 7.1 & .000 & .58 \\
\hline $\mathrm{CMJ}_{\text {loaded }}$ & 4.5 & .100 & .62 & 5.7 & .017 & .82 & 9.8 & .000 & 1.64 \\
\hline FS & 1.4 & 1.000 & .21 & .3 & 1.000 & .03 & 1.7 & 1.000 & .26 \\
\hline
\end{tabular}

\%: porcentaje de cambio; p: p-valor; Es: tamaño del efecto

\section{Discusión}

El principal resultado de este estudio fue que la capacidad de salto vertical incremento significativamente ( $p<.01$ ) un $7.1 \%$ durante el periodo competitivo tras la realización de un entrenamiento de fuerza de con cargas moderadas y un número de repeticiones bajo por serie.

No hemos encontrado estudios en los que se llevara a cabo un análisis del efecto del entrenamiento en jugadores (hombres) de voleibol durante el periodo competitivo con una medición de los resultados en una fecha intermedia del ciclo de entrenamiento, pero sí hemos encontrado un estudio similar llevado a cabo con mujeres jugadoras de voleibol (Newton, Rogers, Volek, Häkkinen, \& Kraemer, 2006) en el que se entrenó durante 11 semanas con un test intermedio a las 7 semanas. Durante las primeras 7 semanas de este estudio se entrenaron los miembros inferiores con «cargas pesadas», aunque lamentablemente no se indica en el artículo cuál es el valor concreto de esas «cargas pesadas» 0 «entrenamiento tradicional de fuerza», y durante las 4 restantes semanas se entrenó con un «entrenamiento balístico», el cual hay que interpretar como un entrenamiento con cargas menores que en las 7 primeras semanas realizadas a alta velocidad y con una carga próxima a aquella con la que se alcanzaba la máxima potencia. El resultado fue que durante las 7 primeras semanas disminuyó el rendimiento en salto de manera significativa, y mejoró en las 4 últimas semanas de manera significativa con respecto al test 2 pero no con respecto al test inicial. La disminución del rendimiento en las primeras 7 semanas es relacionada por estos autores como una probable disminución de la actividad del sistema neuromuscular debido a la excesiva fatiga producida por el entrenamiento habitual técnico-táctico, la competición y un entrenamiento de fuerza con cargas excesivas (cargas pesadas).

Por el contrario, en nuestro estudio las mejoras fueron estadísticamente significativas tras 16 semanas de entrenamiento (7.1\%), habiéndose realizado dicho entrenamiento también conjuntamente con el entrenamiento técnico-táctico y las competiciones. En otro trabajo de las mismas características realizado por nosotros encontramos resultados con una magnitud parecida (5\%) tras seis semanas de entrenamiento (Sánchez-Moreno et al., 2014). Resultados similares a los nuestros se observaron en el estudio de Marques et al (2008) tras 12 semanas de entrenamiento, con una mejora del 3.9\% (cargas 50-75\% 1RM en FS), y en el estudio de Häkkinen et al (1993) tras 10 semanas de entrenamiento en fase competitiva, con una mejora del 4.5\% (cargas $>75 \%$ 1RM en FS). Por el contrario, Voelzke et al (2012) no observaron cambios en CMJ tras cinco semanas de entrenamiento de fuerza (cargas del 85\% 1RM en FS) en ocho jugadores hombres profesionales de voleibol en pretemporada. La diferencia entre los resultados en estos estudios puede venir explicada por la distinta carga de entrenamiento utilizadas, pues en nuestros casos, las cargas pueden considerarse mo- deradas en comparación con las utilizadas en los demás estudios, ya que en nuestros caso no se sobrepasó una carga equivalente al 60-65\% RM en el ejercicio de sentadillas, y los ejercicios de saltos se realizaron con cargas ligeras, por lo que en ambos ejercicios la velocidad de ejecución fue elevada. La superioridad del efecto de los saltos con cargas ligeras (30\% de 1RM) frente a cargas pesadas (80\% de 1RM) también se ha observado en otros estudios (McBride, Triplett-McBride, Davie, \& Newton, 2002). Estos resultados parecen indicar que la especificidad del entrenamiento, que en este caso se manifiesta por la proximidad de la velocidad de los ejercicios de entrenamiento a la velocidad de ejecución del salto vertical, es determinante en el rendimiento.

Además del ejercicio de CMJ, en nuestro estudio también se midió el efecto del entrenamiento sobre la capacidad de salto con cargas adicionales $\left(\mathrm{CMJ}_{\text {loaded }}\right)$ y la sentadilla (FS); se obtuvieron resultados que mostraron un aumento significativo en el $\mathrm{CMJ}_{\text {loaded }}(9.78 \%, \mathrm{p}<.01)$, sin embargo no se observaron cambios significativos en FS (1.66\%, p > .05). Resultados similares hemos observado en un trabajo similar realizado por nosotros (Sánchez-Moreno et al., 2014). Al igual que lo observado en el estudio actual, después de 6 semanas de entrenamiento, se observaron mejoras en el ejercicio de $\mathrm{CMJ}_{\text {loaded }}$ después entrenamiento (5.7\% $\mathrm{p}<.05$ ) sin cambios significativos en FS (4.5\%, $\mathrm{p}=.07$ ). Habitualmente, se mejora en mayor medida la fuerza ante carga altas que con cargas bajas, porque la fuerza máxima, manifestada ante altas cargas, tiene un mayor margen de mejora que la velocidad absoluta de acortamiento muscular (velocidad sin cargas). Sin embargo, en nuestro caso la tendencia fue la opuesta. Es probable que la especificidad del entrenamiento, determinada por la velocidad de ejecución, haya tenido más influencia sobre aquellos ejercicios que se desarrollan a velocidades altas (CMJ y $\mathrm{CMJ}_{\text {loaded }}$ ) que sobre la fuerza máxima ante cargas altas. Por tanto, si tenemos en cuenta que el objetivo prioritario del trabajo fue mejorar el salto sin cargas (CMJ), y que este tipo de rendimiento es el específico y más determinante desde el punto de vista físico en un jugador de voleibol, las cargas de entrenamiento aplicadas en el actual trabajo parecen ser bastante adecuadas para el objetivo que se pretendía.

Marques y col. (2008), observo una mejora del 13\% en el ejercicio de FS tras 12 semanas de entrenamiento. Esta mejora puede estar explicada por la mayor intensidad máxima utilizada en este estudio, que llegó aproximadamente al 75\% de la RM. Estos mismos autores observaron una mejora del $12 \%$ en el ejercicio de $\mathrm{CMJ}_{\text {loaded }}$ tras 12 semanas de entrenamiento. Esta mayor mejora a baja velocidad, propia de una sentadilla con cargas máximas, puede explicar en mayor medida los cambios de velocidad con cargas medias (saltos con cargas), pues cuanto mayor es la carga desplazada, mayor es la varianza de la velocidad explicada por la fuerza máxima (Verkhoshanski, 1986). Sin embargo, estas mayores cargas tuvieron menor efecto en el CMJ (3.9\%) que en nuestro estudio (7.1\%), lo que sugiere que una mayor carga no necesariamente produce mejores resultados sobre acciones a alta velocidad ni presenta mayor relación con el cambio producido después del entrenamiento.

\begin{tabular}{|c|c|c|c|c|c|c|}
\hline \multirow{2}{*}{$\begin{array}{l}\text { Estudios } \\
\text { Nosotros (2015) }\end{array}$} & \multirow{2}{*}{\begin{tabular}{|c|}
$\begin{array}{c}\text { Duración } \\
\text { (semanas) }\end{array}$ \\
$8+8$
\end{tabular}} & \multicolumn{2}{|c|}{ Mejora (\%) } & \multirow{2}{*}{$\begin{array}{c}\text { Genero } \\
\mathrm{H}\end{array}$} & \multirow{2}{*}{$\begin{array}{c}\begin{array}{c}\text { I. Max sentadilla } \\
\text { (\%1RM) }\end{array} \\
65\end{array}$} & \multirow{2}{*}{$\begin{array}{c}\begin{array}{c}\text { I. Max CMJloaded } \\
(\% \mathrm{MP})\end{array} \\
80 \\
\end{array}$} \\
\hline & & 5,3 & 7,1 & & & \\
\hline Nosotros (2014a) & 6 & --- & 5,0 & $\mathrm{H}$ & 60 & 80 \\
\hline Nosotros (2014b) & 8 & --- & 3,4 & $\mathrm{H}$ & 65 & 80 \\
\hline Häkkinen (1993) & 9 & $-\ldots$ & 4,5 & M & $>75$ & n.e \\
\hline Newton y col. (2006) & $7+4$ & $-5,4$ & 5,3 & M & n.e & n.e \\
\hline Marques y col. (2008) & 12 & --- & 3,9 & M & 75 & 100 \\
\hline
\end{tabular}

Estos resultados parecen indicar una vez más que la especificidad del entrenamiento, que en este caso se manifiesta por la proximidad de la velocidad de los ejercicios de entrenamiento a la velocidad deejecución del salto vertical, juega un papel importante en el rendimiento. No obstante, aunque todas estas sugerencias son plausibles, es probable que la tendencia a la disminución o la estabilidad de los resultados pueda venir explicada por el hecho de que un ciclo de una duración superior a las 6-8 semanas no aporte mejoras en sujetos entrenados. Por tanto, es probable que un descanso de entrenamiento de fuerza de una semana antes de iniciar el siguiente ciclo pudiera ser una buena medida para 
permitir nuevas mejoras. Todas estas posibles explicaciones vuelven a poner de manifiesto que es necesario seguir estudiando acerca de cuál es la combinación de cargas y tiempos de entrenamiento más adecuados para permitir una mayor mejora y para mantener esas mejoras durante todo la fase competitiva.

\section{Conclusiones y aplicaciones prácticas}

Nuestros resultados sugieren que el uso de cargas moderadas podría ser suficiente para mejorar el rendimiento del salto vertical, ya que en nuestro estudio no fue superada una carga equivalente al 65\% de 1RM en el FS, y los ejercicios de saltos con cargas fueron realizadas con cargas moderadas. De ello se podría desprender que no es necesario realizar una fase de altas cargas (velocidad media-baja) antes de aplicar cargas ligeras (alta velocidad) para conseguir mejoras en fuerza y velocidad en ejercicios realizados a alta velocidad, tal como se ha venido aplicando tradicionalmente en sujetos altamente entrenados. Estos resultados parecen indicar que la especificidad del entrenamiento, que en este caso se manifiesta por la proximidad de la velocidad de los ejercicios de entrenamiento a la velocidad de ejecución del salto vertical, es determinante en el rendimiento.

Es necesario seguir estudiando acerca de cuál es la combinación de cargas y tiempos de entrenamiento más adecuados para permitir una mayor mejora y para mantener esas mejoras durante toda la fase competitiva en jugadores de voleibol profesionales.

\section{Agradecimientos}

Los autores agradecen al Centro de Investigación en Rendimiento Físico y Deportivo de la Universidad Pablo de Olavide y al equipo de voleibol, Cajasol-Juvasa Equimo, su colaboración en el desarrollo de este estudio. No se han utilizado fuentes de financiación para el desarrollo de dicho estudio. Los autores no tienen conflictos de intereses que estén directamente relacionados con el contenido de este trabajo.

\section{Referencias}

Forthomme, Bénédicte, Croisier, Jean-Louis, Ciccarone, Guido, Crielaard, Jean-Michel, \& Cloes, Marc. (2005). Factors correlated with volleyball spike velocity. The American journal of sports medicine, 33(10), 1513-1519.

Franco-Márquez, F, Rodríguez-Rosell, D, González-Suárez, JM, ParejaBlanco, F, Mora-Custodio, R, Yañez-García, JM, \& González-Badillo, JJ. (2015). Effects of Combined Resistance Training and Plyometrics on Physical Performance in Young Soccer Players. International journal of sports medicine.

Fry, Andrew C, Kraemer, William J, Weseman, Cheryl A, Conroy, Brian P, Gordon, Scott E, Hoffman, Jay R, \& Maresh, Carl M. (1991). The Effects of an Off-season Strength and Conditioning Program on Starters and Non-starters in Women's Intercollegiate Volleyball. The Journal of Strength \& Conditioning Research, 5(4), 174-181.

González-Badillo, Juan J, Pareja-Blanco, Fernando, Rodríguez-Rosell, David, Abad-Herencia, José L, del Ojo-López, Juan J, \& Sánchez-Medina, Luis. (2015). Effects of Velocity-Based Resistance Training on Young Soccer Players of Different Ages. The Journal of Strength \& Conditioning Research, 29(5), 1329-1338.

González-Badillo, Juan J, \& Sánchez-Medina, L. (2010). Movement velocity as a measure of loading intensity in resistance training. Int $J$ Sports Med, 31, 347-352.

González-Ravé, José M, Arija, Alfredo, \& Clemente-Suarez, Vicente. (2011). Seasonal changes in jump performance and body composition in women volleyball players. The Journal of Strength \& Conditioning Research, 25(6), 1492-1501.

Häkkinen, K. (1993). Changes in physical fitness profile in female volleyball players during the competitive season. The Journal of sports medicine and physical fitness, 33(3), 223-232.

Hedges, L. V., \& Olkin, I. (1985). Statistical Methods for Meta-Analysis, New York: Academic Press.
Izquierdo, Mikel, Ibanez, Javier, González-Badillo, Juan José, Häkkinen, Keijo, Ratamess, Nicholas A, Kraemer, William J, . . . Asiain, Xabier. (2006). Differential effects of strength training leading to failure versus not to failure on hormonal responses, strength, and muscle power gains. Journal of Applied Physiology, 100(5), 1647-1656.

Lidor, Ronnie, \& Ziv, Gal. (2010). Physical characteristics and physiological attributes of adolescent volleyball players-a review. Pediatr Exerc Sci, 22(1), 114-134.

López-Segovia, Manuel, Andrés, José M Palao, \& González-Badillo, Juan J. (2010). Effect of 4 months of training on aerobic power, strength, and acceleration in two under-19 soccer teams. The Journal of Strength \& Conditioning Research, 24(10), 2705-2714.

Marques, Mário C, Van Den Tillaar, Roland, Vescovi, Jason D, \& GonzálezBadillo, Juan José. (2008). Changes in strength and power performance in elite senior female professional volleyball players during the in-season: a case study. The Journal of Strength \& Conditioning Research, 22(4), 1147-1155.

McBride, Jeffrey M, Triplett-McBride, Travis, Davie, Allan, \& Newton, Robert U. (2002). The effect of heavy-vs. light-load jump squats on the development of strength, power, and speed. The Journal of Strength \& Conditioning Research, 16(1), 75-82.

Newton, Robert U, Rogers, Ryan A, Volek, Jeff S, Häkkinen, Keijo, \& Kraemer, William J. (2006). Four weeks of optimal load ballistic resistance training at the end of season attenuates declining jump performance of women volleyball players. The Journal of Strength \& Conditioning Research, 20(4), 955-961.

Pareja-Blanco, F, Rodríguez-Rosell, D, Sánchez-Medina, L, Gorostiaga, EM, \& González-Badillo, JJ. (2014). Effect of movement velocity during resistance training on neuromuscular performance. Int J Sports Med, 35(11), 916-924.

Rhea, M. R. (2004). Determining the magnitude of treatment effects in strength training research through the use of the effect size. Journal of Strength and Conditioning Research, 18, 918-920.

Sáez Sáez de Villarreal, Eduardo, Izquierdo, Mikel, \& Gonzalez Badillo, Juan José. (2011). Enhancing jump performance after combined vs. maximal power, heavy-resistance, and plyometric training alone. The Journal of Strength \& Conditioning Research, 25(12), 32743281.

Sanchez-Medina, L, Perez, CE, \& Gonzalez-Badillo, JJ. (2010). Importance of the propulsive phase in strength assessment. Int $J$ Sports Med, 31(2), 123-129.

Sanchez-Medina, Luis, \& González-Badillo, Juan José. (2011). Velocity loss as an indicator of neuromuscular fatigue during resistance training. Med Sci Sports Exerc, 43(9), 1725-1734.

Sánchez-Moreno, Miguel ,García-Asencio, Carlos, \& González-Badillo, Juan José (2014). The effects of short-term resistance program on vertical jump ability in elite male volleyball players during the competition season. RETOS. Nuevas Tendencias en Educación Física, Deporte y Recreación(26), 153-156.

Sheppard, Jeremy M, Chapman, Dale W, Gough, Clare, McGuigan, Michael R, \& Newton, Robert U. (2009). Twelve-month training-induced changes in elite international volleyball players. The Journal of Strength \& Conditioning Research, 23(7), 2096-2101.

Stanganelli, Luiz Cláudio Reeberg, Dourado, Antonio Carlos, Oncken, Percy, Mançan, Sérgio, \& da Costa, Silvano César. (2008). Adaptations on jump capacity in Brazilian volleyball players prior to the under19 World Championship. The Journal of Strength \& Conditioning Research, 22(3), 741-749.

Trajkovic, Nebojša, Milanovic, Zoran, Sporis, Goran, Milic, Vladan, \& Stankovic, Ratko. (2012). The effects of 6 weeks of preseason skillbased conditioning on physical performance in male volleyball players. The Journal of Strength \& Conditioning Research, 26(6), 14751480.

Verkhoshanski, Y. (1986). Fundamentals of special strength-training in sport: Sportivny Press.

Voelzke, Mathias, Stutzig, Norman, Thorhauer, Hans-Alexander, \& Granacher, Urs. (2012). Promoting lower extremity strength in elite volleyball players: Effects of two combined training methods. Journal of Science and Medicine in Sport, 15(5), 457-462. 